\title{
Die kardiopulmonale Reanimation von Kindern (Paediatric Life Support)
}

\author{
Christoph Eich, Bernd Landsleitner
}

\section{Übersicht}

Grundlegende Aspekte des

kindlichen Atem-Kreislauf-

Stillstands

Änderungen 2015

Lebensrettende Basismaßnahmen

bei Kindern

\section{0}

\section{Grundlegende Aspekte des kindlichen Atem-Kreislauf- Stillstands}

\section{Extrem zeitkritisch}

Ein kindlicher Atem-Kreislauf-Stillstand ist ein seltenes, aber besonders zeitkritisches Ereignis. Ein wichtiges Ziel der Leitlinien zu den lebensrettenden Maßnahmen bei Kindern (Paediatric Life Support, PLS) ist es, das kritisch kranke oder verletzte Kind rechtzeitig zu erkennen und zu behandeln, um den drohenden Atem-Kreislauf-Stillstand möglichst zu verhindern.

\section{- Geringe Inzidenz}

Exakte Zahlen zur Inzidenz der kardiopulmonalen Reanimation bei Kindern sind für Deutschland derzeit nicht erhältlich. Laut Daten aus den USA liegen sie etwa 20-mal niedriger als bei Erwachsenen [1]. Berücksichtigt man zusätzlich lokale Daten und Daten des Deutschen Reanimationsregisters, können damit für Deutschland etwa 3000 - 4000 kindliche Reanimationen pro Jahr kalkuliert werden, davon ca. ein Viertel außerklinisch [2,3]. In einer regionalen präklinischen Untersuchung wurde für einen durchschnittlich häufig tätigen Notarzt eine statistische Häufigkeit von einer Kinderreanimation pro 9 Jahre berechnet [4]. Lokale Erhebungen und Umfragen zeigen, dass selbst in spezialisierten Kinderzentren mit einer relativ geringen
Der Beitrag wurde erstmals veröffentlicht in Notfallmedizin up2date 2016; 11: 51-67.

Inzidenz von oft $<1$ Reanimation pro Monat gerechnet wird $[5,6]$.

\section{- Geringe kindernotfallmedizinische Erfahrung}

Spezialisierte Kindernotarztsysteme werden nur in wenigen deutschen Ballungszentren vorgehalten, z. B. in der Stadt München [7,8]. Neugeborenennotarztsysteme wiederum dienen i. d. R. ausschließlich der Erstversorgung von Neugeborenen und deren Transport. Die Einsatzradien und damit die Eintreffzeiten von Kindernotarztsystemen sind aufgrund ihrer zentralisierten Standorte deutlich größer als die der regulären Notarztsysteme. Eine beliebige Expansion der Kindernotarztsysteme in die Fläche ist wegen der beschränkten Anzahl geeigneter Kinderkliniken, den limitierten personellen Ressourcen und nicht zuletzt aufgrund unzureichender Finanzierungsmöglichkeiten jedoch nicht möglich.

- Somit werden auch in Deutschland die Mehrzahl der prä- und innerklinischen Kindernotfälle initial von Nicht-Spezialisten mit oft geringer kindernotfallmedizinischer Erfahrung versorgt $[9,10]$.

Dabei zeigt sich, dass aufgrund von Angst und Unsicherheit notfallmedizinische oder gar Reanimationsmaßnahmen bei Kindern häufig unterbleiben oder verzögert eingeleitet werden [11]. 
Die Reanimation bei Kindern und Säuglingen ist ein seltenes Ereignis. Die primäre Versorgung übernehmen dabei überwiegend Nicht-Spezialisten.

\section{- Schwierige Datenlage}

Es gibt nach wie vor nur wenige klinische Studien zur kardiopulmonalen Reanimation von Kindern, insbesondere keine randomisiert-kontrollierten Studien. So beruhen wesentliche Empfehlungen auf der wissenschaftlichen Evidenz von Tierversuchen und Simulationsmodellen, auf Daten aus Kinderanästhesie und Kinderintensivmedizin sowie auf der Extrapolierung von Erwachsenendaten.

\section{- Starke Vereinheitlichung und Angleichung der Leitlinien}

Um Erinnerbarkeit, Implementierung und Anwendung der Leitlinien zu erleichtern, wurde großer Wert auf eine Vereinfachung der pädiatrischen Leitlinien und eine möglichst weitgehende Angleichung an die Erwachsenenleitlinien gelegt [12]. Kinder unterscheiden sich stark hinsichtlich Alter, Größe und Gewicht. In den pädiatrischen Leitlinien des ERC werden jedoch, nicht zuletzt aus didaktischen Gründen, nur 3 Altersgruppen definiert:

- Neugeborene (hier: unmittelbar postpartal im Kreißsaal; Versorgung gemäß Neonatal Life Support, NLS)

- Säuglinge (<1 Jahr)

- Kinder ( $\geq 1$ Jahr bis zum sichtbaren Beginn der Pubertät)

Ab dem sichtbaren Beginn der Pubertät werden Kinder wie Erwachsene behandelt.

\section{- Vielfältige Ätiologie}

Die Ätiologie kindlicher Atem-Kreislauf-Stillstände ist wesentlich inhomogener als bei Erwachsenen. Primär kardiale Ursachen mit plötzlich einsetzendem AtemKreislauf-Stillstand und primärem Kammerflimmern (ventricular fibrillation, VF) bzw. pulsloser Kammertachykardie (VT) sind bei Kindern ohne Herzvitium bzw. außerhalb kinderkardiologisch/-kardiochirurgischer Zentren selten [13]. Im Vordergrund stehen respiratorische Störungen (Atemwegsinfekte, Asthma, Fremdkörperaspiration, Ertrinken) und zirkulatorische Störungen (Dehydratation, Traumata, Verbrennungen, Sepsis, Anaphylaxie), oft in Kombination [14]. Auch akute neurologische Erkrankungen können sekundär durch Asphyxie mit Myokardhypoxie und konsekutiver Bradykardie zum Atem-Kreislauf-Stillstand führen (z.B. Epilepsie, Vergiftungen, Schädel-Hirn-Trauma, Meningitis, Enzephalitis) [15]. Beim kindlichen Atem-Kreis-
lauf-Stillstand liegen daher initial meist eine pulslose elektrische Aktivität (PEA) oder eine Asystolie vor [16].

Dieser besonderen ätiologisch-pathophysiologischen Konstellation bei Kindern trägt der Begriff „AtemKreislauf-Stillstand“ Rechnung. Aus respiratorischer und zirkulatorischer Insuffizienz resultiert eine Myokardhypoxie, erkennbar an einer progredienten Bradykardie. Wird die kausale Ursache nicht effektiv beseitigt, kommt es zum Atem-Kreislauf-Stillstand.

Bei Kindern resultiert der Atem-Kreislauf-Stillstand i.d.R. aus einer kardiopulmonalen Dekompensation aufgrund einer über einen längeren Zeitraum bestehenden, respiratorischen und/oder zirkulatorischen Störung und vergleichsweise selten aus einem kardialen Ereignis.

\section{Rechtzeitiges Erkennen und Behandeln}

Durch die oft über Stunden oder Tage vorliegende, protrahierte und ausgeprägte globale Hypoxie, Hyperkapnie und Azidose bei Eintritt des Atem-KreislaufStillstands ist, insbesondere präklinisch, das Reanimationsergebnis bei Kindern meist schlecht [16]. Die wirkungsvollste Strategie, eine permanente neurologische Schädigung oder den Tod des Kindes zu verhindern, ist daher die Vermeidung des Atem-Kreislauf-Stillstands durch rechtzeitiges Erkennen und effektive Behandlung des kritisch kranken oder verletzten Kindes, inklusive effektiver Ersthelfermaßnahmen. Darauf wird beim Paediatric Life Support besonders großes Augenmerk gelegt.

\section{Änderungen 2015}

\section{- Ziele der Leitlinienüberarbeitung}

Die jeweils neuen Leitlinien zur Reanimation sollen den aktuellen Stand der Wissenschaft berücksichtigen und nur dann Änderungen etablierter Verfahrensweisen empfehlen, wenn diese ein nachgewiesen besseres Outcome haben (Evidenz). Diese Evidenz ist insgesamt sehr limitiert. Als gesichert gilt z. B., dass bei Kindern mit nicht kardial bedingtem Atem-Kreislauf-Stillstand eine Ersthelferreanimation mit Beatmung ein deutlich besseres primäres (Überleben) und sekundäres (neurologischer Status) Outcome bewirkt, als wenn ausschließlich Thoraxkompressionen durchgeführt werden [17].

Änderungen bestehender Empfehlungen benötigen erheblichen Zeitaufwand, um trainiert und flächendeckend in die tägliche Praxis implementiert zu wer- 
den [18]. Insofern ist eine Änderung per se gar nicht erstrebenswert. Das zweite entscheidende Kriterium bei der Erstellung der Leitlinien ist die Praktikabilität angesichts sehr unterschiedlicher Strukturen. Dieses Kriterium beinhaltet u. a. die Aspekte Wissensvermittlung, Training, lokale Implementierung und Anwendbarkeit.

\section{CAB-vs. $A B C$-Schema}

Im Rahmen des 2015er CoSTR-Prozesses (CoSTR = Consensus-on-Science-Treatment-Recommendations) des International Liaison Committee on Resuscitation (ILCOR) wurde bestätigt, dass es nach wie vor keine klinischen Studien gibt, die bezüglich Outcome die Basic-Life-Support-Sequenzen „CAB“ (Circulation/Thoraxkompression, Atemwege freimachen, Beatmung) und „ABC“ (Atemwege freimachen, Beatmung, Circulation/Thoraxkompressionen) miteinander vergleichen. Insofern stellte sich die Frage, ob das in Europa langjährig etablierte ABC-Schema beibehalten werden soll. Dies hätte den Vorteil, dass keine Umschulungen erforderlich sind und - zumindest theoretisch - die überwiegend respiratorische Ätiologie des kindlichen Atem-Kreislauf-Stillstands besser berücksichtigt wird. Zur Alternative stand die Neueinführung des CABSchemas auch für die Kinderreanimation, mit dem Vorteil, dass alle Helfer, unabhängig von ihrem Qualifikationsniveau, Patienten aller Altersklassen nach gleicher Sequenz reanimieren können.

Aufgrund der fehlenden klinischen Evidenz haben sich ERC und American Heart Association (AHA) letztlich für die Beibehaltung ihrer 2010 jeweils empfohlenen Sequenzen entschieden: das ERC für $\mathrm{ABC}$ und die $\mathrm{AHA}$ für $\mathrm{CAB}$.

\section{- Konkrete Änderungen in den ERC-Leitlinien}

In den aktuellen ERC-Leitlinien wurden folgende Änderungen / Präzisierungen vorgenommen:

\section{Klassifikation der Helfer. Bei der Durchführung von} Basismaßnahmen (Basic Life Support, BLS) wird de facto zwischen 3 Qualifikationsniveaus der Helfer unterschieden:

- Helfer mit Verpflichtung zur Notfallversorgung von Kindern (z.B. Rettungsdienst, Notaufnahme, Pädiatrie, Anästhesie) wenden die P-BLS-Sequenz an (ABC, 15:2, Kapitel „Lebensrettende Basismaßnahmen bei Kindern“).

- Helfer mit professioneller Verantwortung für Kinder (z. B. Erzieher, Lehrer, Bademeister, Betreuer), aber keiner professionellen PLS-Ausbildung modifizieren die ihnen bekannten BLS-Maßnahmen für Erwach-

\section{Paediatric basic life support}

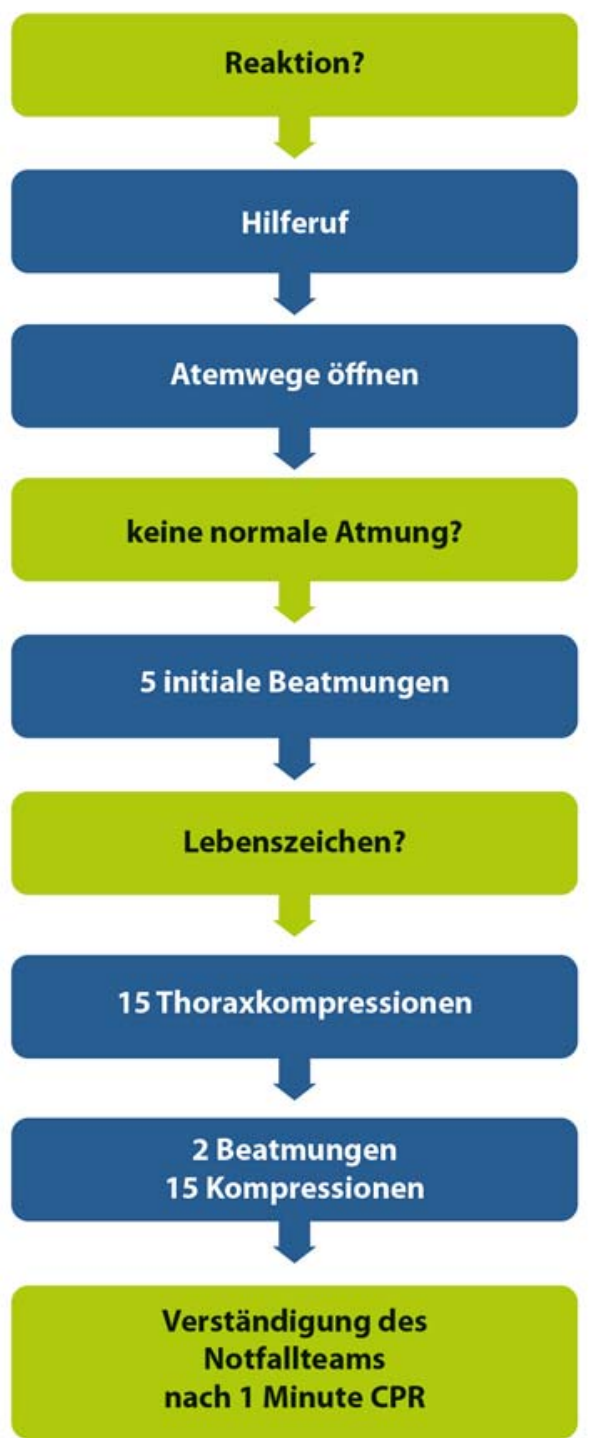

sene: 5 Initialbeatmungen (ABC-Sequenz, 30:2) und 1 min kardiopulmonale Reanimation (cardiopulmonary resuscitation, CPR), bevor sie aktiv Hilfe holen.

- Laienhelfer reanimieren Kinder wie Erwachsene (CAB-Sequenz, 30:2).

Beatmung. Die Dauer eines Beatmungshubes wurde auf $1 \mathrm{~s}$ festgelegt und damit dem Vorgehen bei Erwachsenen angepasst.

Thoraxkompressionen. Der Druckpunkt und die Frequenz von Thoraxkompressionen blieben unverändert, wurden jedoch etwas detaillierter beschrieben: untere
Abb. 1 Basismaßnahmen der kardiopulmonalen Reanimation (cardiopulmonary resuscitation, (PR) beim Kind (Paediatric Basic Life Support, P-BLS). 

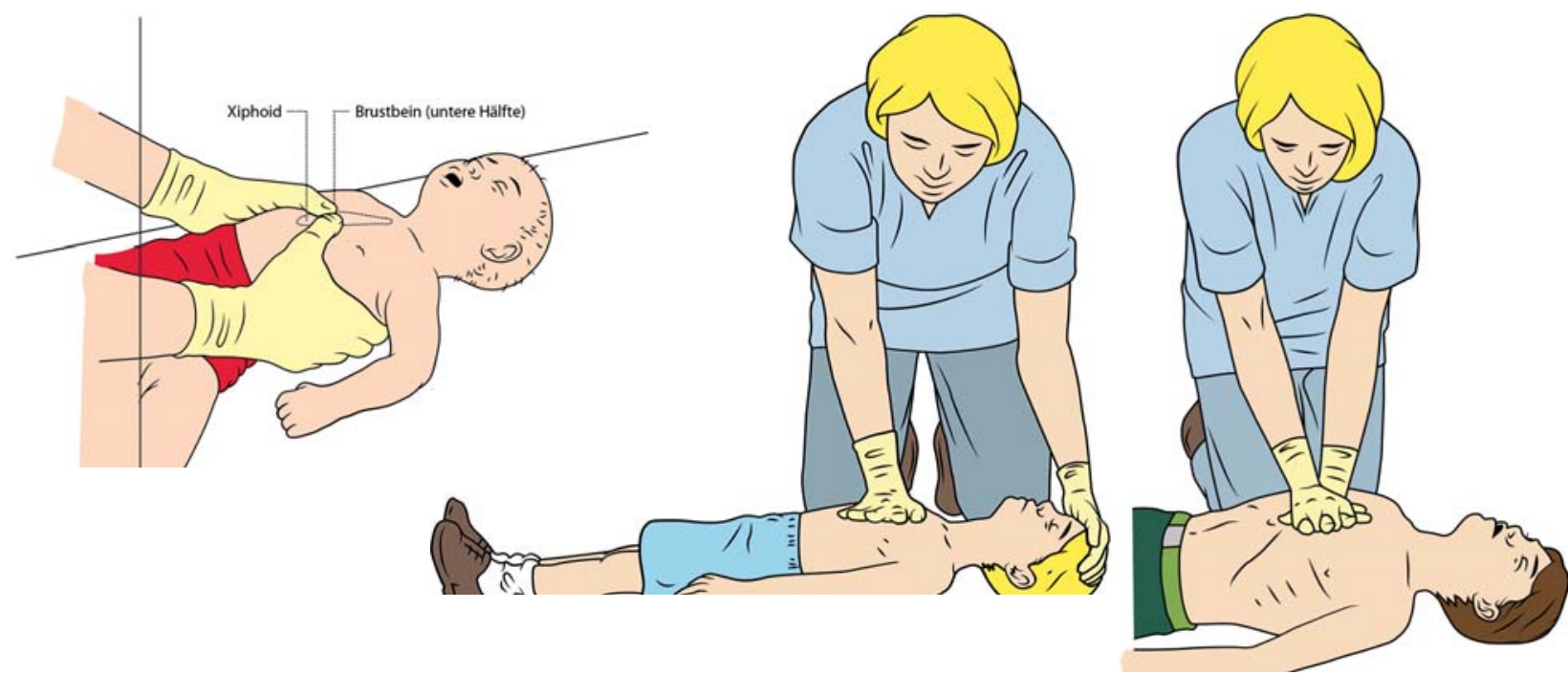

Abb.2 a Thorax-umschließende 2-Daumentechnik beim Säugling; b 1-Handtechnik beim Kind; c 2-Handtechnik beim größeren Kind und Jugendlichen.

Sternumhälfte (=Thoraxmitte), Drucktiefe $1 / 3$ des anterior-posterioren Thoraxdurchmessers (also $4 \mathrm{~cm}$ beim Säugling, $5 \mathrm{~cm}$ beim Kind > 1 Jahr), Kompressionsfrequenz $100-120 / \mathrm{min}$ )

Behandlung kritisch kranker Kinder. Kinder mit Fieber, die keine Zeichen eines septischen Schocks aufweisen, sollten eine zurückhaltende Volumenzufuhr erhalten und regelmäßig reevaluiert werden.

Tachykardie-Algorithmus. Im Tachykardie-Algorithmus wurde die initiale Energiedosis zur Kardioversion einer supraventrikulären Tachykardie auf $1 \mathrm{~J} / \mathrm{kg}$ und die folgende auf $2 \mathrm{~J} / \mathrm{kg}$ festgelegt.

\section{Postreanimationsbehandlung}

- Nach dem Wiedereinsetzen des Spontankreislaufs (return of spontaneous circulation, ROSC) soll Fieber bereits präklinisch vermieden werden.

- Beim zielgerichteten Temperaturmanagement (TTM) nach ROSC sind 2 Konzepte als gleichwertig einzustufen:

- strikte Normothermie $\left(36-37,5^{\circ} \mathrm{C}\right)$ oder

- milde Hypothermie $\left(32-34^{\circ} \mathrm{C}\right)$.

Konsequent vermieden werden müssen in jedem Fall Fieber (Hyperthermie) und schwere Hypothermie $\left(<32^{\circ} \mathrm{C}\right)$.

\section{Lebensrettende Basis- maßnahmen bei Kindern}

\section{- Diagnose „Kreislaufstillstand“}

Die Diagnose „Kreislaufstillstand“ darf nicht durch Pulstasten als einziges Entscheidungskriterium getroffen werden [19]. Vielmehr muss auf Lebenszeichen geachtet werden (Spontanbewegung, Atmen, Husten). Zusätzliche Kriterien für professionelle Helfer sind Pulslosigkeit oder eine Bradykardie (Herzfrequenz $<60 / \mathrm{min}$ ) mit unzureichender Perfusion (u. a. gräulichlivide oder marmorierte Haut, kühle Extremitäten, Rekapillarisierungszeit über dem Sternum >2 - 3s).

\section{- Basismaßahmen}

Die Basismaßnahmen bei Kindern folgen nach wie vor der Sequenz „ABC“. Aufgrund der hohen Wahrscheinlichkeit einer primär respiratorischen Ursache werden bei Kindern 5 initiale Beatmungen durchgeführt.

- Bei einem komatösen Kind, das nicht normal atmet (Cave: Schnappatmung!) und keine Kreislauf- bzw. Lebenszeichen zeigt, sollte umgehend mit der CPR begonnen werden: Thoraxkompressionen und Beatmungen im Verhältnis von 15:2 (Abb. 1 und Abb.2).

Die Basismaßnahmen bei Kindern folgen weiterhin der Sequenz $A B C$ und dem Verhältnis von

Thoraxkompressionen und Beatmungen von 15:2.

\section{Besondere Situationen}

Einzelne professionelle Helfer oder Laienhelfer können alternativ ein Verhältnis von 30:2 anwenden, um die perfusionslosen Intervalle durch die Wechsel zwischen 
Beatmung und Herzdruckmassage („No-Flow-Zeit“) möglichst gering zu halten. Gemäß den Leitlinien sollen alle professionellen Helfer so ausgestattet sein, dass in der BLS-Sequenz unverzüglich 5 Initialbeatmungen appliziert werden können (Maske-Beutel-System griffbereit). Sollte es im Einzelfall zu einer verzögerten Bereitstellung des Maske-Beutel-Systems kommen (z. B. Pflegekraft nachts alleine auf Normalstation, Initialversorgung im Rettungsdienst), so ist die Anwendung einer Erwachsenen-BLS-Sequenz beginnend mit Thoraxkompressionen zu erwägen. Diese Alternative ist in den Leitlinien nicht explizit erwähnt, doch aus pragmatischen Erwägungen mutmaßlich sinnvoller, als ein therapiefreies Intervall.

Im Zweifelsfall und um Verzögerungen zu vermeiden, können Einzelhelfer und Kinder-unerfahrene Helfer Kinder nach dem Erwachsenenalgorithmus reanimieren (Beginn mit Thoraxkompressionen, Verhältnis 30:2).

\section{- Automatisierte externe Defibrillatoren}

Zur Verwendung automatisierter externer Defibrillatoren (AEDs) bei Kindern liegen nur Fallberichte über erfolgreiche Einsätze vor [20,21]. Daraus resultierend werden AEDs für Kinder> 1 Jahr empfohlen. Falls vorhanden, sollten für Kinder von 1 - 8 Jahren Kinder-Pads eingesetzt werden und die Energie sollte nach den Empfehlungen des Herstellers reduziert werden. Der extrem selten erforderliche Einsatz eines AED bei Säuglingen bleibt eine Einzelfallentscheidung. Für Kinder > 8 Jahre können Erwachsenen-AED ohne Einschränkungen verwendet werden.

- Keinesfalls darf der Einsatz eines AED zu Verzögerungen oder vermeidbaren Unterbrechungen der Basismaßnahmen führen.

\section{Maßnahmen bei Fremdkörperaspiration}

Die Aspiration eines Fremdkörpers kann zur Verlegung der Atemwege führen. Hier ist es wichtig, die Situation zu erfassen und die Symptome einzuschätzen (Tab. 1). Der Algorithmus zu den Basismaßnahmen nach Fremdkörperaspiration unterscheidet unverändert 3 Situationen (Abb.3):

- Bei kräftigem Hustenstoß (Tab. 1) und ansprechbarem Kind soll der Patient zum Husten ermuntert und beobachtet werden.

- Ist dagegen der Hustenstoß unzureichend und erschöpft sich der Patient zusehends (Tab. 1), soll der intrathorakale Druck durch jeweils 5 einzelne Schläge auf den Rücken zwischen die Schulterblätter sowie durch 5 Thoraxkompressionen (Säuglinge) bzw.

\section{Tabelle 1}

Daten aus [32].

\section{Zeichen der Fremdkörperverlegung der Atemwege}

allgemeine Zeichen der Fremdkörperverlegung der Atemwege

beobachteter Vorfall

Husten/Ersticken

plötzlicher Beginn

während oder unmittelbar nach dem Spielen mit kleinen Gegenständen bzw. dem Essen

\begin{tabular}{|c|c|}
\hline ineffektives Husten & effektives Husten \\
\hline kann nicht sprechen & $\begin{array}{l}\text { Weinen oder verbale Reaktion } \\
\text { auf Ansprache }\end{array}$ \\
\hline stilles oder leises Husten & lautes Husten \\
\hline kann nicht atmen & kann vor dem Hustenstoß einatmen \\
\hline Zyanose & bewusstseinsklar \\
\hline $\begin{array}{l}\text { sich verschlechternder } \\
\text { Bewusstseinszustand }\end{array}$ & \\
\hline
\end{tabular}

\section{Fremdkörperentfernung beim Kind}

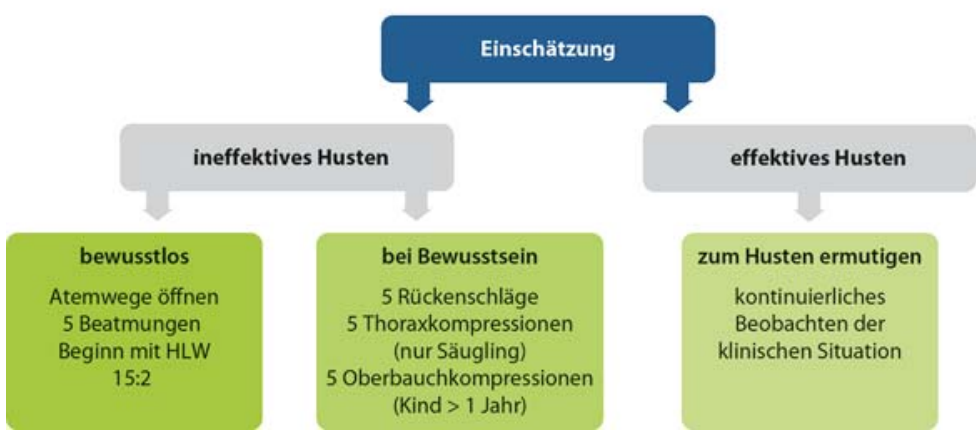

Abb. 3 HLW $=$ Herz-Lungen-Wiederbelebung.

abdominelle Kompressionen (Kinder > 1 Jahr) erhöht werden, um den Fremdkörper auszustoßen.

- Bei Bewusstlosigkeit soll primär die Mundhöhle auf leicht entfernbare Objekte inspiziert werden, bevor dann unverzüglich mit den Basismaßnahmen der Reanimation (Beatmung und Thoraxkompressionen) begonnen wird. 


\section{Erweiterte lebensrettende Maßnahmen bei Kindern}

\section{Frühzeitiges Erkennen und effektives Behandeln des kritisch kranken Kindes}

\section{Pädiatrische Notfälle}

Bei Kindern steht der hypoxische, nicht kardial bedingte Kreislaufstillstand im Vordergrund, und das Outcome nach Reanimation ist tendenziell schlechter als bei Erwachsenen. Daher liegt bei Kindern der Fokus besonders auf der Vermeidung des Atem-KreislaufStillstands. Mehr als 80 \% aller pädiatrischen Notfälle lassen sich in 3 große Gruppen gliedern $[2,10]$ :

- akute Atemstörungen,

- akute neurologische Störungen (v. a. Krampfanfälle) und

- Traumata.

Da auch bei den beiden letztgenannten Gruppen Atembzw. Atemwegsstörungen häufig sind, spielt das Atemwegsmanagement bei pädiatrischen Notfällen generell eine zentrale Rolle.

Abb. 4 Maskenbeatmung beim Säugling. a 1-HandMaskengriff; b 2-Hand-EsmarchMaskengriff.
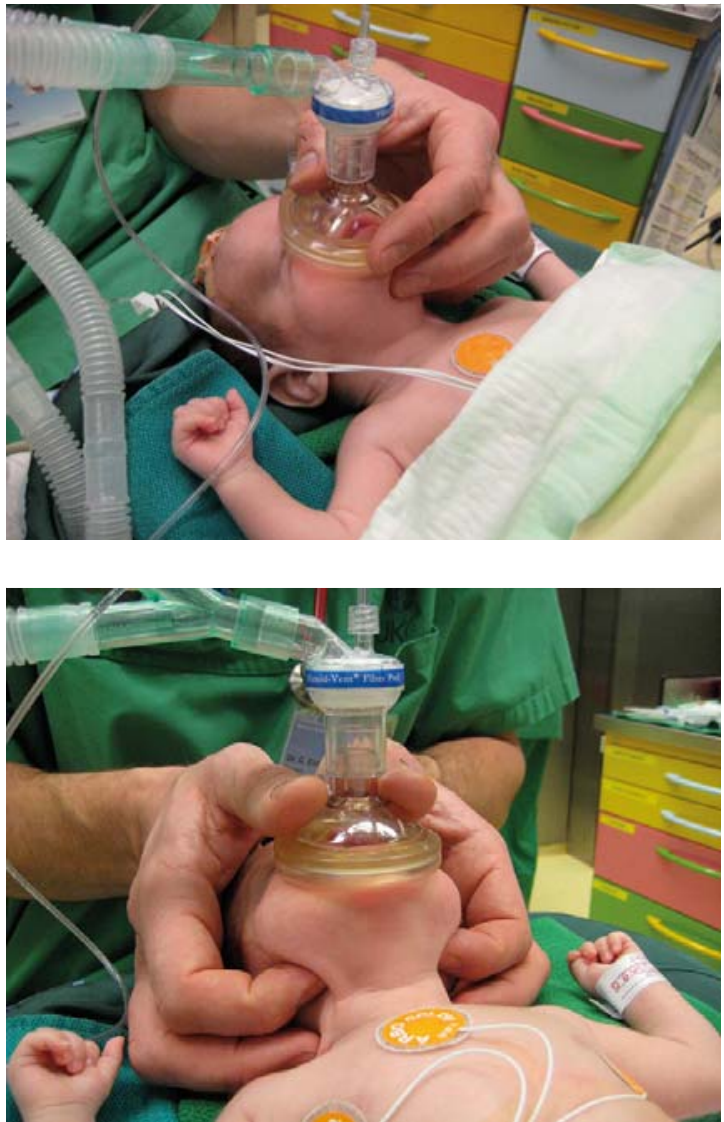

\section{Strukturiertes Vorgehen nach $A B C D E$}

Die strukturierte Beurteilung und Behandlung des kritisch kranken oder verletzten Kindes soll nach dem bekannten ABCDE-Schema erfolgen und hat zum Ziel, drohendes respiratorisches oder zirkulatorisches Versagen frühzeitig zu erkennen. Die Bewertung und Behandlung der Punkte „D“ (Defizit/neurologischer Status) und „E“ (Exploration) ist nicht Gegenstand der Leitlinien, wird aber u. a. in den „European Paediatric Advanced Life Support“-Kursen (EPALS) gelehrt.

\section{Grenzwerte definieren}

Für die frühzeitige Alarmierung eines innerklinischen Notfallteams (Medical emergency Team, MET) kann es hilfreich sein, für die erfassten Vitalparameter Grenzwerte im Sinne eines „Paediatric Early Warning Score“ (PEWS) zu definieren [22]. Dabei müssen jeweils die altersentsprechenden Normalwerte berücksichtigt werden.

\section{Klinische Erstabschätzung}

Das sog. „Pädiatrische Dreieck“ (Paediatric Assessment Triangle) erlaubt eine einfache und rasche klinische Ersteinschätzung potenziell kranker oder verletzter Kinder innerhalb von $<30 \mathrm{~s}$ ohne technische Hilfsmittel [23]. Der Helfer konzentriert sich dabei auf

- den Gesamteindruck (u. a. Bewusstsein, Muskeltonus, Verhalten),

- die Atemarbeit (u. a. Einziehungen, pathologische Atemgeräusche) und

- die Durchblutung der Haut (u. a. Blässe, Marmorierung, Zyanose).

\section{Weiteres Vorgehen}

Die weitere strukturierte Evaluation und Behandlung folgt dann dem ABCDE-Schema (beurteilen, verändern, erneut beurteilen).

Zu Beginn wird das Bewusstsein kontrolliert: Ist das Kind wach, somnolent, soporös, komatös? (AVPU = alert, response to verbal or painful stimulus, unresponsive)

\section{A: Atemwege und B: (Be-)Atmung}

\section{Beurteilung}

Die Notfalluntersuchung beginnt mit der Beurteilung des Atemwegs und der (Be-)Atmung: 
A: Atemweg

- offen?

- in Gefahr?

- verlegt?

\section{B: (Be-)Atmung}

- Atemfrequenz (zu schnell, zu langsam?)

- Atemarbeit (z. B. Einziehungen, Nasenflügeln?)

- pathologische Atemgeräusche (z.B. Stridor, Giemen, Stöhnen oder [einseitig] fehlendes Atemgeräusch?)

- vermindertes Atemzugvolumen (z. B. flache Atemzüge, schwacher Atemstoß, geringe Thoraxexkursionen, gemessene Hyperkapnie)

- Zeichen der Hypoxämie (z. B. Zyanose, schlechte pulsoxymetrische $\mathrm{O}_{2}$-Sättigung)

- Zeichen der hypoxischen Kreislaufdepression (zunächst Tachykardie, später Bradykardie, Blässe, Bewusstseinsstörung)

\section{Sicherung}

Zur Sicherung von Atemweg und Atmung stehen u. a. folgende Maßnahmen zur Verfügung, die bei frühzeitigem Einsatz meist den Eintritt des Atem-KreislaufStillstands verhindern können:

- Freimachen der Atemwege und Unterstützung der Atmung durch adäquate Lagerung (Nacken-/Schulterrolle, Seitenlage oder Oberkörper hoch) und Mobilisierung von Unterkiefer und Zunge (Hals überstrecken/Kinn anheben oder Esmarch-Handgriff), ggf. vorsichtig absaugen.

- bei offenen Atemwegen: $\mathrm{O}_{2}$-Gabe, initial in max. Konzentration, später Dosistitration nach Stabilisierung

- Inhalation von $\beta$-Mimetika bei V. a. bronchiale Obstruktion; Inhalation von Adrenalin bei V. a. laryngo-tracheale oder unklare Obstruktion

- assistierte Beatmung (möglichst mit PEEP) bei Hypoventilation (Abb.4); bei entsprechender Erfahrung und Ausstattung auch nicht-invasive Ventilation (NIV)

- engmaschige Überwachung von Klinik, $\mathrm{SpO}_{2}$ und etCO ${ }_{2}$ (Kapnografie auch unter Spontanatmung möglich und sinnvoll)

- Invasive Manipulationen an den Atemwegen sollten möglichst vermieden, Sonden und Absaugkatheter zurückhaltend und nur bei eindeutiger Indikation eingesetzt werden.

- Die komplette Ausstattung zur kontrollierten MaskeBeutel-Beatmung (Plan A) und mind. eine alternative Technik zur Atemwegssicherung (Plan B) sollten vorbereitet werden.

- Falls die Einleitung einer Allgemeinanästhesie und die invasive Atemwegssicherung zum sicheren
Repertoire des Notfallteams gehören, sollten diese frühzeitig vorbereitet und das Vorgehen abgesprochen werden.

\section{C: Circulation (Kreislauf)}

\section{- Symptome}

Die Zeichen der Kreislaufdepression können gerade in der Initialphase der Notfallversorgung eines Kindes übersehen werden, weil sie initial häufig unauffällig sind und oft von der Stressreaktion maskiert werden: - verlängerte Rekapillarisierungszeit (capillary refill time) als frühes Zeichen: Nagelbett, Stirn oder Sternum werden für $5 \mathrm{~s}$ komprimiert und danach beobachtet: Kehrt die (rosige) Durchblutung in max. $2 \mathrm{~s}$ zurück, so ist die Mikrozirkulation mutmaßlich normal.

- Tachykardie, unmittelbar vor kardialer Dekompensation auch Bradykardie (altersabhängige Normalwerte beachten)

- Blutdruckabfall (i.d. R. spätes Zeichen, altersabhängige Normalwerte und adäquate Manschettengröße beachten)

- schwache oder fehlende periphere Pulse; ggf. auch nicht ableitbares Pulsoxymeter-Signal.

- Hinweise auf vermindertes (z. B. Anamnese, Unfallmechanismus) oder erhöhtes Intravasalvolumen (Vorlast)

- verminderte Urinausscheidung

\section{- Maßnahmen}

Bei Anzeichen einer Kreislaufstörung stehen folgende Maßnahmen zur Verfügung:

- Gefäßzugang legen, ggf. intraossär (i. o.)

- Volumenbolus von $20 \mathrm{ml} / \mathrm{kg}$ einer isotonen Lösung (idealerweise: bilanzierte Vollelektrolytlösung [VEL]), außer in folgende Situationen:

- primär kardiale Funktionsstörungen (z. B. Kardiomyopathie, Myokarditis): Hier darf nur eine vorsichtige Volumengabe erfolgen.

- fieberhafte Erkrankungen ohne Zeichen einer Kreislaufstörung: Hier sollte auf einen Volumenbolus zunächst verzichtet und das Kind engmaschig reevaluiert werden.

- Ergänzend zur Volumengabe kann auch eine Katecholamintherapie erforderlich sein. Bestehen Gründe für eine Volumenrestriktion, so ist dies ggf. die erste Maßnahme zur Kreislaufstabilisierung. 

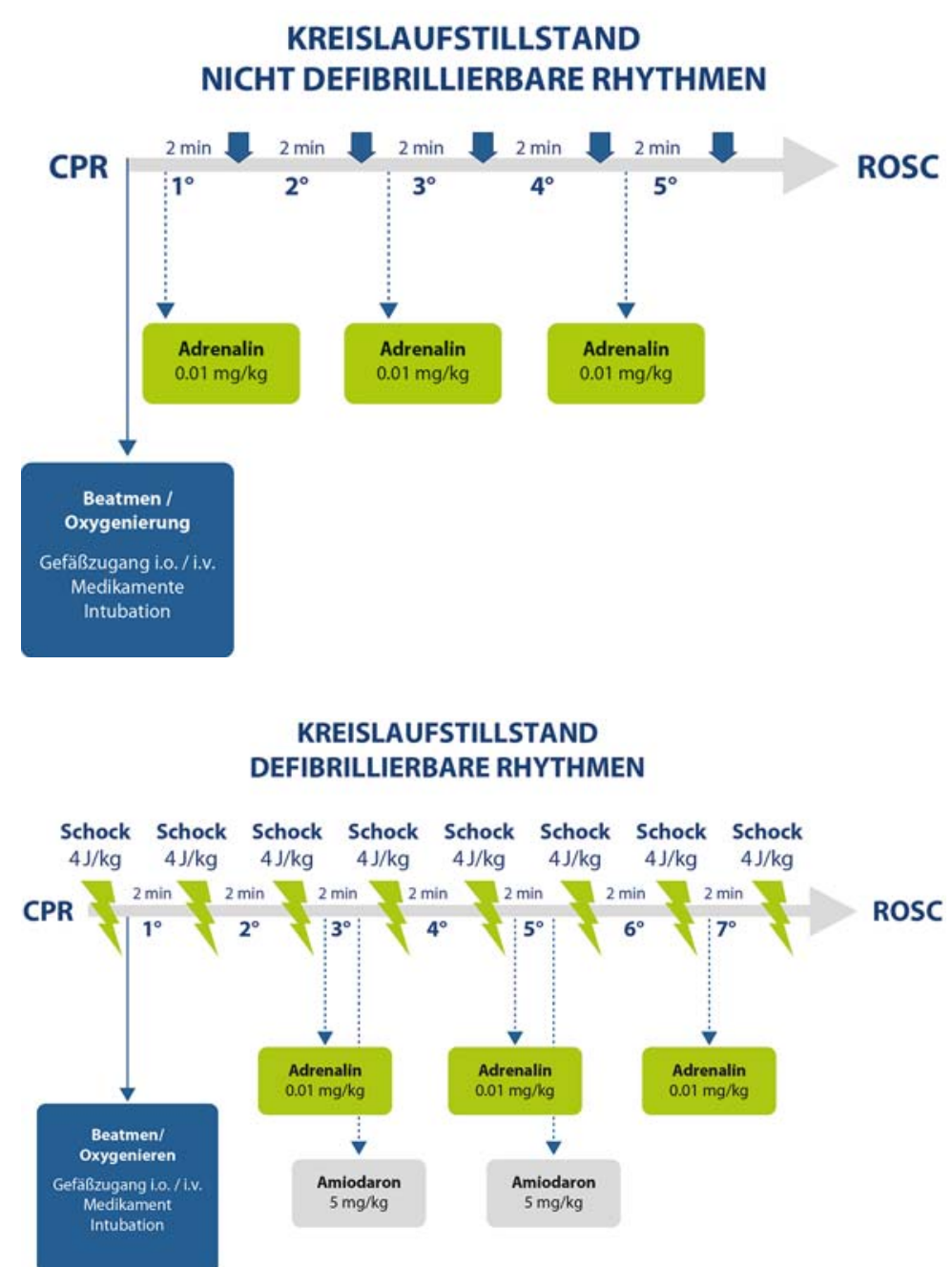

Abb.5a Kreislaufstillstand bei pulsloser elektrischer Aktivität und Asystolie; b Kreislaufstillstand bei Kammerflimmern und pulsloser Kammertachykardie. CPR = cardiopulmonary resuscitation; ROSC = return of spontaneous circulation.

Das frühzeitige Erkennen und die effektive Notfalltherapie des kritisch kranken oder verletzten Kindes hat oberste Priorität. Dadurch kann der AtemKreislauf-Stillstand häufig verhindert werden.

\section{Erweiterte Maßnahmen beim Atem- Kreislauf-Stillstand}

\section{Nicht defibrillierbare Rhythmen}

Da der Atem-Kreislauf-Stillstand im Kindesalter meist hypoxisch bedingt ist, sind nicht defibrillierbare Arrestrhythmen (Asystolie/PEA) die Regel. Hier beginnen die erweiterten Reanimationsmaßnahmen mit der Applikation von Adrenalin $(0,01 \mathrm{mg} / \mathrm{kg}=0,1 \mathrm{ml} / \mathrm{kg}$ einer $1: 10000$ Lösung) in einem Dosisintervall von $3-5 \mathrm{~min}$ bzw. nach jedem 2 . Zyklus ( 1 Zyklus $=2 \mathrm{~min}=10 \times 15$ Thoraxkompressionen plus $10 \times 2$ Beatmungen). Dazu wird, wenn innerhalb von 1 min kein sicherer venöser Zugang etabliert werden kann, ein i.o. Zugang gelegt Abb.5a).

\section{Defibrillierbare Rhythmen}

Bei Kammerflimmern bzw. pulsloser Kammertachykardie wird ein initialer Defibrillationsversuch (4J/kg; mono- oder biphasisch) empfohlen (Abb. $\mathbf{5} \mathbf{b}$ und Abb.6). Anschließend werden unabhängig vom resultierenden Rhythmus die Thoraxkompressionen und die Beatmung (CPR) über 2 min fortgeführt, bevor ein erneuter Defibrillationsversuch unternommen wird. Adrenalin wird hier erst nach der 3. Defibrillation appliziert und alle 3-5 min wiederholt. Ebenfalls nach der 3. Defibrillation wird Amiodaron ( $5 \mathrm{mg} / \mathrm{kg}$ ) gegeben, ggf. mit einmaliger Wiederholung nach der 5.Defibrillation in gleicher Dosis.

\section{- Potenziell reversible Ursachen}

Für den primären Reanimationserfolg (ROSC) bzw. zu dessen Sicherung sind das Erkennen und die Behandlung potenziell reversibler Ursachen des Atem-Kreislauf-Stillstands bei Kindern besonders wichtig.Diese sind erinnerbar mit Hilfe des Akronyms „4 Hs und HITS":

- Hypoxie und Hypovolämie (beide mit besonders hoher Relevanz bei Kindern), Hypothermie, Hypound Hyperkaliämie sowie

- Herzbeuteltamponade, Intoxikation, Thromboembolie (koronar und pulmonal) und Spannungspneumothorax.

Abb. 6 zeigt den P-ALS-Algorithmus (Paediatric Advanced Life Support). Im Folgenden werden die Maßnahmen im Einzelnen beschrieben.

\section{A: Atemwege}

\section{Maske-Beutel-Beatmung}

Die Maske-Beutel-Beatmung ist in der Initialphase der Reanimation die Standardmethode zur kontrollierten Beatmung (Abb.4). Ihre Effektivität kann durch adäquate Lagerung (inkl. Einsatz einer Nacken-/Schulterrolle bei Säuglingen), optimal passende Masken, gute Abdichtung und ggf. durch den Einsatz eines orooder nasopharyngealen Rachentubus in korrekter Insertionstiefe verbessert werden (Abb.7).

\section{Supraglottische Atemwege}

Bei prolongierter Reanimation oder Beatmung bzw. bei ineffektiver Maske-Beutel-Beatmung sollte möglichst ein anderes Verfahren gewählt werden. Supraglotti- 
sche Atemwege haben den Vorteil einer steilen Lernkurve und relativ geringer Komplikationsraten. Eine Visualisierung der Atemwege ist zu ihrer Platzierung nicht erforderlich. Der supraglottische Atemweg mit der mit Abstand größten Anwendungserfahrung in der anästhesiologischen Routineversorgung und in der Notfallmedizin ist die Larynxmaske [24].

\section{Endotracheale Intubation}

Wie bei Erwachsenen soll die endotracheale Intubation auch bei Kindern, insbesondere präklinisch, nur bei entsprechender Expertise des Anwenders und nach strenger Indikation erfolgen. Denn bei Unerfahrenen verzögern sich regelhaft die höher priorisierten Maßnahmen der kardiopulmonalen Reanimation (Herzdruckmassage, Defibrillation) und es kann zu schwerwiegenden Sekundärkomplikationen kommen [25,26]. Die Verwendung blockbarer (gecuffter) Endotrachealtuben wird bei Kindern jenseits des Neugeborenenalters als sinnvoll und sicher angesehen.

\section{B: Beatmung}

\section{- Überwachung mittels Kapnografie}

Der mutmaßliche Hauptvorteil einer Atemwegssicherung mittels supraglottischem Atemweg oder Intubation liegt in der dann möglichen, ununterbrochenen Herzdruckmassage beim Kreislaufstillstand und damit in der Minimierung der No-Flow-Zeit [27]. Allerdings besteht dabei die Gefahr der Hyperventilation mit konsekutiver, vasokonstriktorisch bedingter zerebraler Minderperfusion.

- Der Einsatz der Kapnografie zur Lagekontrolle und Überwachung der Atemwegssicherung, zur Früherkennung des wieder einsetzenden Kreislaufs (ROSC) und zur Vermeidung einer Hyperventilation nach ROSC ist obligat.

\section{C: Circulation}

\section{- Flüssigkeitstherapie und Medikation}

- Flüssigkeitstherapie: Bei Verdacht auf einen bei Kindern häufig vorliegenden Volumenmangel wird ein Bolus einer plasmaisotonen kristalloiden Lösung von $20 \mathrm{ml} / \mathrm{kg}$ appliziert (ideal: balanzierte VEL) .

- Adrenalin wird bei allen Formen des Atem-KreislaufStillstands im Abstand von 3-5 min und in einer Dosierung von $0,01 \mathrm{mg} / \mathrm{kg}(=0,1 \mathrm{ml} / \mathrm{kg}$ einer $1: 10000$-Lösung) verabreicht.

\section{Paediatric Advanced Life Support}
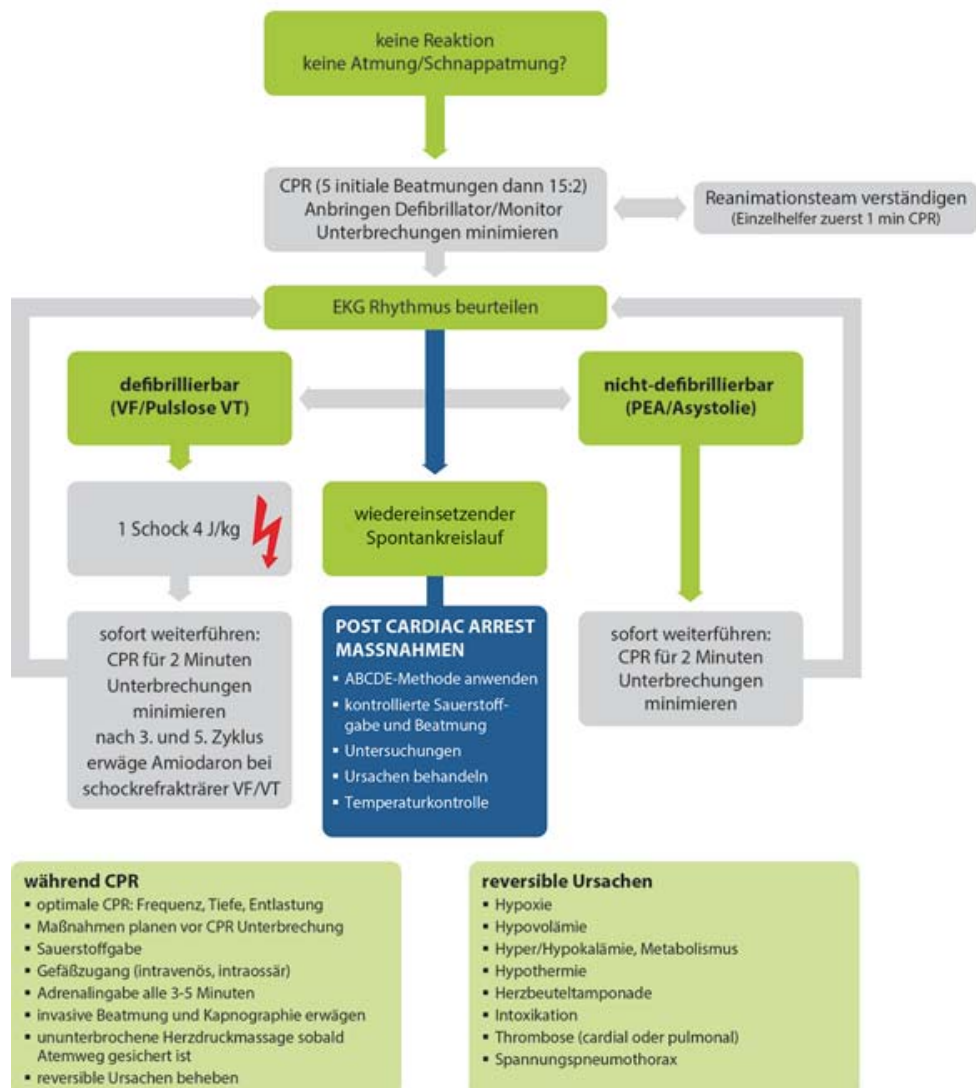

reversible Ursachen$$
\text { - Hypoxie }
$$

- Hyper/Hypokalāmie, Metabolismus

- Hypothermie

- Herzbeuteltamponade

- Intoxikation

- Thrombose (cardial oder pulmonat)

- Spannungspneumothorax

Abb. 6 Erweiterte lebensrettende Maßnahmen der kardiopulmonalen Reanimation (cardiopulmonary resuscitation, CPR) beim Kind (Paediatric Advanced Life Support, P-ALS). $\mathrm{PEA}=$ pulslose elektrische Aktivität; $\mathrm{VF}=$ ventrikuläre Fibrillation; $\mathrm{VT}=$ ventrikuläre Tachykardie.

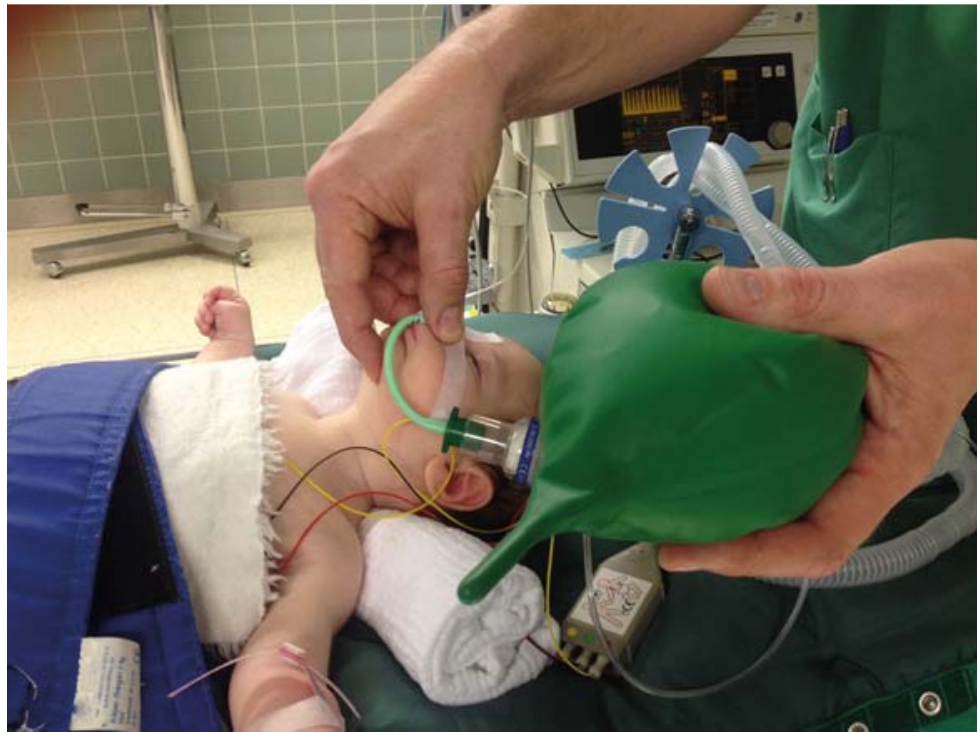

Abb.7 Beatmung eines Säuglings via nasopharyngealem Rachentubus (Mund und kontralaterales Nasenloch verschlossen. 


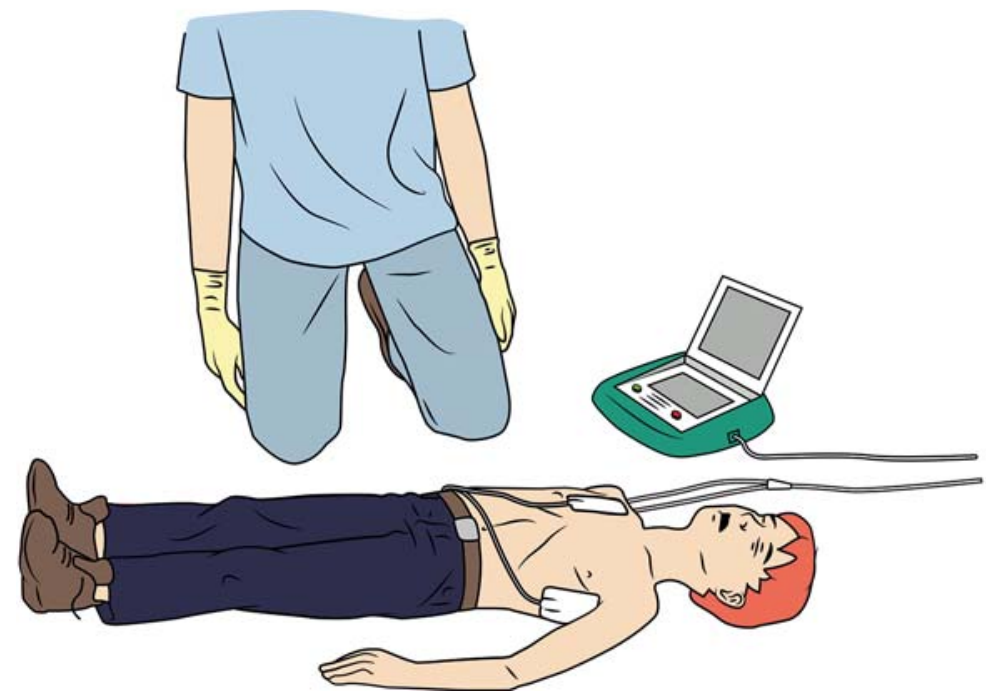

Abb. 8 Defibrillation beim Kind. Bildnachweis: Maconochie IK, Bingham R, Eich C et al. Lebensrettende Maßnahmen bei Kindern („paediatric life support“). Notfall + Rettungsmedizin 2015; 18: 932 -963. (c) European Resuscitation Council.

- Amiodaron wird bei defibrillierbaren Rhythmen nach der 3. Defibrillation in einer Dosierung von $5 \mathrm{mg} / \mathrm{kg}$ gegeben und bei Erfolglosigkeit einmalig nach dem 5. Schock wiederholt.

- Atropin wird adjuvant nur bei Bradykardien eingesetzt, wenn die Ursache in einem erhöhten Vagotonus oder einer Cholinergika-Intoxikation vermutet wird. Die übliche vagolytische Dosis beträgt $0,02 \mathrm{mg} / \mathrm{kg}$ (min.100 $\mu \mathrm{g} / \mathrm{kg}$ ).

- Kalzium wird nicht routinemäßig bei der Reanimation eingesetzt. Nur bei Hypokalziämie, Intoxikation mit Kalziumantagonisten, Hypermagnesämie und Hyperkaliämie kann die Gabe erwogen werden (z. B. 0,25-0,5 ml/kg Kalziumglukonat $10 \%$ ).

- Glukose wird nur bei nachgewiesener Hypoglykämie fraktioniert gegeben (z. B. 1 -2,5 ml/kg Glukose 20 \%). Glukosehaltige, insbesondere hypotone Infusionslösungen müssen aus Sicherheitsgründen unbedingt vermieden werden.
- Natriumbikarbonat wird nicht routinemäßig, sondern nur nach vorliegender Blutgasanalyse eingesetzt. Die „blinde“ Gabe sollte nur bei Intoxikation mit trizyklischen Antidepressiva erwogen werden.

- Zu Vasopressin oder Terlipressin kann weder eine positive noch eine negative Empfehlung gegeben werden.

\section{Besonderheiten der manuellen Defibrillation}

Bei der manuellen Defibrillation sind folgende Dinge zu beachten:

- Die Energiedosis beträgt $4 \mathrm{~J} / \mathrm{kg}$.

- Selbstklebende Pads sind sicherer und effektiver und sollten daher bevorzugt verwendet werden. Sie sollten für Säuglinge $<10 \mathrm{~kg}$ einen Durchmesser von $4,5 \mathrm{~cm}$ haben, für ältere Kinder $8-12 \mathrm{~cm}$.

- Die richtige Pad-Position ist rechts unterhalb der Klavikula und links axillar (Abb.8). Falls die Pads zu groß für den kindlichen Thorax sind und die Gefahr eines direkten Spannungsübertritts besteht, kann auch anterior-posterior geklebt werden: links parasternal und links unterhalb des Schulterblatts.

Bei eingetretenem Atem-Kreislauf-Stillstand kommt es v. a. auf die sofortige und hochqualitative Anwendung von Beatmung und Thoraxkompressionen an.

\section{Versorgung nach Wiederherstellung eines Spontankreislaufs}

\section{Beatmung und Temperaturmanagement}

Im Hinblick auf eine mögliche Beeinträchtigung der zerebralen Perfusion soll eine (akzidentelle) Hyperventilation bei der Reanimation von Kindern unbedingt vermieden werden. Bei gesichertem Atemweg wird eine Beatmungsfrequenz von $12-20 / \mathrm{min}$ empfohlen; angestrebt wird ein normaler arterieller $\mathrm{CO}_{2}$-Partialdruck.

\section{Fazit}

Im Vergleich zu Erwachsenen stehen bei Kindern, hypoxisch bedingte Kreislaufstillstände aufgrund akuter oder subakuter respiratorischer Störungen mit konsekutiver pulsloser elektrischer Aktivität (PEA) oder Asystolie im Vordergrund. Die aktuellen Leitlinien 2015 des European Resuscitation Council (ERC) zu den lebensrettenden Maßnahmen bei Kindern legen daher besonderes Augenmerk auf das frühzeitige Erkennen und die effektive Behandlung des kritisch kranken oder verletzten Kindes, also auf die Vermeidung des Atem-Kreislauf-Stillstands, sowie auf den frühzeitigen Beginn von Ersthelfermaßnahmen. Bei den Algorithmen zu den Basis- und erweiterten lebensrettenden Maßnahmen hat es im Vergleich zu 2010 keine grundlegenden Änderungen gegeben. Die Sequenz ABC (Atemwege, Beatmung, Circulation) wurde beibehalten. Bei eingetretenem Atem-Kreislauf-Stillstand kommt es v. a. auf die sofortige und hochqualitative Anwendung von Thoraxkompressionen und Beatmung im Verhältnis $15: 2$ an. 
Zur therapeutischen Hypothermie bzw. zum zielgerichteten Temperaturmanagement (TTM) von anhaltend komatösen Kindern nach erfolgreicher Reanimation (ROSC) gibt es nur wenige Daten. Basierend auf Studien bei Neugeborenen mit perinataler Hypoxie, Erwachsenendaten und einer aktuellen Kinderstudie gibt es

2 Möglichkeiten [28-30]:

- therapeutische Hypothermie mit Kühlung auf $32-34^{\circ} \mathrm{C}$ über mind. $24 \mathrm{~h}$ oder

- strikt kontrollierte Normothermie $\left(36-37,5^{\circ} \mathrm{C}\right)$.

Sicher vermieden werden müssen in jedem Fall Fieber (Hyperthermie) und eine schwere Hypothermie $\left(<32{ }^{\circ} \mathrm{C}\right)$.

\section{Kindernotfallmedizinische Kursformate}

\section{Kursspektrum}

Zur notfallmedizinischen Versorgung von Kindern bieten der European Resuscitation Council (ERC) und der German Resuscitation Council (GRC) 3 standardisierte Kursformate an:

- den 2- bis 3-tägigen EPALS-Kurs (European Paediatric Advanced Life Support) für Ärzte, Pflege- und Rettungsdienstpersonal, die regelmäßig mit der Versorgung kritisch kranker oder verletzter Kinder betraut sind;

- den 1-tägigen EPILS-Kurs (European Paediatric Immediate Life Support) für die unmittelbare Erstversorgung von Kindern mit manifestem oder drohendem Atem-Kreislauf-Stillstand und

- den 1-tägigen NLS-Kurs (Newborn Life Support) für die unmittelbar postpartale Neugeborenenversorgung.
Darüber hinaus gibt es das Notfallmedizinische Curriculum Kinderheilkunde der Deutschen Interdisziplinären Vereinigung für Intensiv und Notfallmedizin (DIVI), weitergehende, Simulator-basierte Kindernotfallkurse (z. B. PaedSim) sowie zahlreiche lokal adaptierte kindernotfallmedizinische Kursformate.

\section{Über die Autoren}

\section{Prof. Dr. med. Christoph Eich}

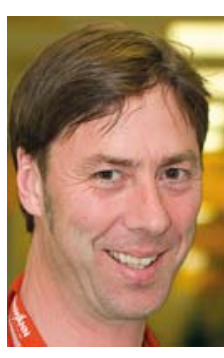

Prof. Dr. med. Christoph Eich ist

Chefarzt der Abteilung Anästhesie, Kinderintensiv- und Notfallmedizin im Kinder- und Jugendkrankenhaus AUF DER BULT in Hannover. Er ist Mitglied der ILCOR-Arbeitsgruppe Paediatric Life Support (PLS), Ko-autor der PLS-Leitlinien des European Resuscitiation Council (ERC) 2010

und 2015, Mitglied des Exekutivkomitees des Deutschen Wiederbelebungsrats (GRC) und 2. Sprecher des Wissenschaftlichen Arbeitskreises Kinderanästhesie der DGAI.

\section{Dr. med. Bernd Landsleitner}

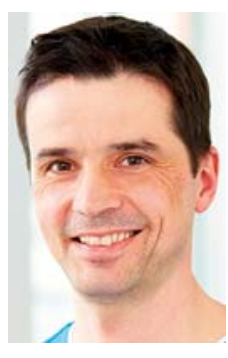

Dr. med. Bernd Landsleitner ist Leitender Oberarzt der Abteilung für Anästhesie und Intensivmedizin in der Cnopfschen Kinderklinik/Klinik Hallerwiese in Nürnberg. Er ist aktiver Notarzt, European-ResuscitationCouncil-Instruktor (ALS/EPALS) und Mitglied in der Arbeitsgruppe Kindernotfallmedizin des Wissenschaft-

lichen Arbeitskreises Kinderanästhesie der DGAI.

\section{Kernaussagen}

- Die Reanimation bei Kindern und Säuglingen ist ein seltenes Ereignis. Die primäre Versorgung erfolgt dabei überwiegend durch Nicht-Spezialisten.

- Der plötzliche Kollaps aufgrund eines kardialen Ereignisses ist bei Kindern selten. In der Regel führt eine über einen längeren Zeitraum bestehende, respiratorische und/oder zirkulatorische Störung zu kardiopulmonaler Dekompensation und schließlich zum hypoxisch bedingten Atem-Kreislauf-Stillstand.
- Das frühzeitige Erkennen und die effektive Notfalltherapie des kritisch kranken oder verletzten Kindes hat oberste Priorität. Dadurch kann der Atem-KreislaufStillstand oft verhindert werden.

- Bei eingetretenem Atem-Kreislauf-Stillstand kommt es v. a. auf die sofortige und hochqualitative Anwendung von Beatmung und Thoraxkompressionen an.

- Die Empfehlungen des European Resuscitation Council (ERC) zu den Basismaßnahmen bei Kindern mit der Sequenz
„ABC“ und dem Verhältnis von Thoraxkompressionen und Beatmungen von $15: 2$ blieben unverändert.

- Im Zweifelsfall und um Verzögerungen zu vermeiden können Einzel- und Kinder-unerfahrene Helfer Kinder nach dem Erwachsenenalgorithmus reanimieren (Beginn mit Thoraxkompressionen, Verhältnis $30: 2$ ).

- Die Empfehlungen des ERC zu den erweiterten lebensrettenden Maßnahmen (ALS) bei Kindern blieben ebenfalls weitestgehend unverändert. 


\section{Korrespondenzadresse}

Prof. Dr. med. Christoph Eich

Kinder- und Jugendkrankenhaus Auf der Bult

Abteliung Anästhesie, Kinderintensiv- und Notfallmedizin

Janusz-Korczak-Allee 12

30173 Hannover

E-Mail: eich@hka.de

Interessenkonflikt: Die Autoren erklären, dass keine Interessenkonflikte vorliegen.

\section{Literatur}

1 Topjian AA, Berg RA, Nadkarni VM. Pediatric cardiopulmonary resuscitation: advances in science, techniques, and outcomes. Pediatrics 2008; 122: 1086 - 1098

2 Eich C, Russo SG, Heuer JF et al. Characteristics of out-of-hospital paediatric emergencies attended by ambulance- and helicopter-based emergency physicians. Resuscitation 2009; 80: $888-892$

3 Gräsner JT, Meybohm P, Fischer M et al. A national resuscitation registry of out-of-hospital cardiac in Germany - a pilot study. Resuscitation 2009; 80: 199-203

4 Bernhard M, Helm M, Luiz T et al. Pädiatrische Notfälle in der prähospitalen Notfallmedizin - Implikationen für die Notarztqualifikation. Notfall Rettungsmed 2011; 14: 554-566

5 Hoffmann F, Müller M, Gräsner JT et al. Retrospektive Umfrage zur Häufigkeit von Reanimationen und Hypothermiebehandlungen auf pädiatrischen Intensivstationen in Deutschland. Anästhlntensivmed 2011; 52

6 Tibballs J, Kinney S. Reduction of hospital mortality and of preventable cardiac arrest and death on introduction of a pediatric medical emergency team. PediatrCrit Care Med 2009; 10: $306-312$

7 Bayerl R. Das Münchner Kindernotarztsystem: 1553 Einsätze aus zwei der vier Kinderkliniken in den Jahren 1998-2000 [Dissertation]. München: Ludwig-Maximilians-Universität München, Medizinische Fakultät; 2007

8 Stenke CP. Der Münchner Kindernotarzt: 3667 Kindernotarzteinsätze der Jahre 1998-2001 untersucht an zwei der vier beteiligten Kliniken [Dissertation]. München: Ludwig-Maximilians-Universität München, Medizinische Fakultät; 2004

9 Gries A, Zink W, Bernhard M et al. Einsatzrealität im Notarztdienst. Notfall Rettungsmed 2005; 8: 391 - 398

10 Nagele P, Kroesen G. Kindernotfälle im Notarztdienst. Eine epidemiologische Studie am Notarztwagen Innsbruck. Anaesthesist 2000; 49: 725-731

11 Ballnus S, Möller JC, Friedrich HJ. Evaluation der dokumentierten notärztlichen Versorgung von Kindern im Einzugsgebiet des Universitätsklinikums Lübeck. Der Notarzt 2002; 18 : $187-191$
12 Maconochie IK, Bingham R, Eich C et al. European Resuscitation Council Guidelines for Resuscitation 2015: Section 6. Paediatric life support. Resuscitation 2015; 95: 223- 248

13 Johnson MA, Grahan BJ, Haukoos JS et al. Demographics, bystander CPR, and AED use in out-of-hospital pediatric arrests. Resuscitation 2014; 85: 920 - 926

14 Atkins DL, Everson-Stewart S, Sears GK et al. Epidemiology and outcomes from out-of-hospital cardiac arrest in children: the Resuscitation Outcomes Consortium Epistry-Cardiac Arrest. Circulation 2009; 119: 1484-1491

15 European Resuscitation Council. Hrsg. Paediatric Life Support (EPLS). Course Manual. Based upon the ERC Guidelines 2010. 4. ed. Edegem: European Resuscitaion Council; 2011

16 Herlitz J, Engdahl J, Svensson L et al. Characteristics and outcome among children suffering from out of hospital cardiacarrest in Sweden. Resuscitation 2005; 64: 37 - 40

17 Kitamura T, Iwami T, Kawamura T et al. Conventional and chest-compression-only cardiopulmonary resuscitation by bystanders for children who have out-of-hospital cardiac arrests: a prospective, nationwide, population-based cohort study. Lancet 2010; 375: 1347 - 1354

18 Berdowski J, Schmohl A, Tijssen JGP, Koster RW. Time needed for a regional emergency medical system to implement resuscitation Guidelines 2005 -The Netherlands experience. Resuscitation 2009; 80: 1336-1341

19 Tibballs J, Weeranatna $C$. The influence of time on the accuracy of healthcare personnel to diagnose paediatric cardiac arrest by pulse palpation. Resuscitation 2010; 81: 671-675

20 Gurnett CA, Atkins DL. Successful use of a biphasic waveform automated external defibrillator in a high-risk child. Am J Cardiol 2000; 86: 1051 - 1053

21 Perkins GD, Handley AJ, Koster KW et al. European Resuscitation Council Guidelines for Resuscitation 2015: Section 2. Adult Basic Life Support and Automated External Defibrillation. Resuscitation 2015; 95: 81 - 98

22 Panesar R, Polikoff LA, Harris D et al. Characteristics and outcomes of pediatric rapid response teams before and after mandatory triggering by an elevated Pediatric Early Warning System (PEWS) score. HospPediatr 2014; 4: 135-140

23 Dieckmann RA, Brownstein D, Gausche-Hill M. The Pediatric Assessment Triangle: a novel approach for the rapid evaluation of children. Pediatric Emergency Care 2010; 26: 312-315

24 Keil J, Jung P, Schiele A et al. Interdiszplinär konsentierte Stellungnahme zum Atemwegsmanagement mit supraglottischen Atemwegshilfen in der Kindernotfallmedizin Larynxmaske ist State-of-the-art. Anästhesist [Epub ahead of print] 2015

25 Gausche M, Lewis RJ, Stratton SJ et al. Effect of out-of-hospital pediatric endotracheal intubation on survival and neurological outcome: a controlled clinical trial. JAMA 2000; 283: 783 790

26 Timmermann A, Russo SG, Eich C et al. The out-of-hospital esophageal and endobronchial intubations performed by emergency physicians. AnesthAnalg 2007; 104: 481-483

27 Berg RA, Sanders AB, Kern KB et al. Adverse hemodynamic effects of interrupting chest compressions for rescue breathing during cardiopulmonary resuscitation for ventricular fibrillation cardiac arrest. Circulation 2001; 104: 2465 - 2470 
28 Bistritz JF, Horton LM, Smaldone A. Therapeutic hypothermia in children after cardiac arrest: a systematic review and metaanalysis. PediatrEmerg Care 2015; 31: 296-303

29 Moler FW, Silverstein FS, Holubkov R et al. Therapeutic hypothermia after out-of-hospital cardiac arrest in children. N Engl J Med 2015; 372: 1898 - 1908

30 Scholefield BR, Morris KP, Duncan HP et al. Evolution, safety and efficacy of targeted temperature management after pediatric cardiac arrest. Resuscitation 2015; 92: 19-25
31 Weiss M, Dullenkopf A, Fischer JE et al. Prospective randomized controlled multi-centre trial of cuffed or uncuffed endotracheal tubes in small children. Br J Anaesth 2009; 103: 867 873

32 Maconochie IK, Bingham R, Eich C et al. Lebensrettende Maßnahmen bei Kindern ("paediatriclife support") - Kapitel 6 der Leitlinien zur Reanimation (Deutsche Übersetzung durch Burda G et al.). Notfall Rettungsmed 2015; 18: $932-963$ 


\section{CME॰thieme.de}

\section{CME-Fragen}

\section{CME-Teilnahme}

- Viel Erfolg bei lhrer CME-Teilnahme unter http://cme.thieme.de

- Diese Fortbildungseinheit ist 12 Monate online für eine CME-Teilnahme verfügbar.

- Sollten Sie Fragen zur Online-Teilnahme haben, unter http://cme.thieme.de/hilfe finden Sie eine ausführliche Anleitung.

\section{1}

Welche Aussage zum kindlichen Atem-Kreislauf-Stillstand ist richtig?
A Die Inzidenz ist deutlich geringer als bei Erwachsenen.
B Die führende Ursache sind Herzrhythmusstörungen.
C Die führende Ursache sind Traumata.
D Ein Vollzeit-Notfallmediziner erlebt diesen Notfall mehrmals im Jahr.
E Er wird präklinisch in Deutschland meist vom Kinder-Notarzt versorgt.

\section{2}

Welche Altersgruppen werden in den ERC-Leitlinien zur KinderReanimation von 2015 definiert?
A Neugeborenene (bis 4 Wochen), Säuglinge (1 - 12 Monate), Kinder (1 - 6 Jahre, Schulkinder (7 - 13 Jahre), Heranwachsende (14-20 Jahre)

B Neugeborene (unmittelbar postpartal), Säuglinge (< 1 Jahr), Kinder (1 Jahr bis zum sichtbaren Beginn der Pubertät)

C Frühgeborene, Reifgeborene, Kinder $<6$ Jahre, Kinder $>6$ Jahre

D Keine Altersdifferenzierung 0-14 Jahre

E Säuglinge, (0-12 Monate), Kleinkinder (1 - 3 Jahre)

\section{3}

Welche Aussage zum Basic-LifeSupport bei Kindern ist richtig?

A Es gibt harte Evidenz für das $A B C$ gegenüber dem $C A B-S c h e m a$.

B Helfer dürfen keinesfalls BLS-Maßnahmen für Erwachsene bei Kindern anwenden.

C Die Drucktiefe bei der Thoraxkompression darf $2 \mathrm{~cm}$ bei Kleinkindern nicht überschreiten.

D Entscheidend ist die unterbrechungsfreie Anwendung qualitativ hochwertiger Basismaßnahmen.

E Ein Beatmungshub dauert mindestens 2 Sekunden, um eine Magenüberblähung zu verhindern.

4

Welche Aussage zu den Maßnahmen bei Fremdkörperaspiration ist richtig?
A Bei ansprechbarem Kind mit effektivem Hustenstoß sollen möglichst frühzeitig abdominelle Kompressionen (Heimlich-Handgriff) angewendet werden.

B Bei bewusstlosem Kind werden zunächst 5 Schläge auf den Rücken verabreicht und dann bei ausbleibendem Erfolg 5 abdominelle Kompressionen (Heimlich-Handgriff).

C Das ansprechbare Kind mit kräftigem Hustenstoß wird lediglich zum Husten ermuntert und beobachtet.

D Das ansprechbare Kind mit kräftigem Hustenstoß wird mit 5 Rückenschlägen und ggf. 5 abdominelle Kompressionen (Heimlich-Handgriff) versorgt.

E Abdominelle Kompressionen (Heimlich-Handgriff) werden auch bei Säuglingen angewendet.
5

Welche Notfallsituation ist bei Kindern selten?
A Akutes Koronarsyndrom
B Akute Atemstörungen
C Akute neurologische Störungen
D Akuter Atemwegsinfekt
E Traumata 


\section{CME-Fragen}

Die kardiopulmonale Reanimation von Kindern (Paediatric Life Support)

\section{6}

Was wird bei Kindernotfällen mit dem Akronym AVPU beschrieben?
A Die Bewusstseinslage
B Die Rekapillisierungszeit
C Die respiratorische Situation
D Der maximal tolerable Blutverlust
E Die Sequenz zur präklinischen Polytraumaversorgung

\section{7}

Welche Aussage zur Durchführung der Beutel-Maske-Beatmung beim Säugling ist falsch?
A Der Kopf wird mithilfe einer Nacken-/Schulterrollle in Neutralposition gelagert.

B Die Zwei-Hand-Methode verbessert die Dichtigkeit der Maske und die Effektivität des Esmarch-Handgriffs.

C Die Beatmung sollte mit moderatem Druck erfolgen; eine eben sichtbare Thoraxexkursion ist ausreichend.

D Beim nicht-komatösen bzw. - narkotisierten Kind muss immer mit einer funktionellen Atemwegsverlegung gerechnet werden.

E Die invasive Ventilation über einen Endotrachealtubus zeigt einen deutlichen Überlebensvorteil gegenüber der Beutel-Maske-Beatmung.

\section{8}

Welche Aussage zur Applikation von Medikamenten während der Reanimation von Kindern ist falsch?
A Die endobronchiale Applikation wird nicht mehr empfohlen.

B Die intraossäre Kanüle ist in den meisten Fällen der erste Applikationsweg.

C Adrenalin wird intravenös in einer Dosierung von $0,01 \mathrm{mg} / \mathrm{kg}$ appliziert.

D Adrenalin wird intraossär in einer Dosierung von $0,1 \mathrm{mg} / \mathrm{kg}$ appliziert.

E Amiodaron wird intraossär in einer Dosierung von $5 \mathrm{mg} / \mathrm{kg}$ appliziert.

\section{9}

Was unterscheidet den Paediatric Advanced Life Support (PALS-) Algorithmus vom Erwachsenen (ALS-) Algorithmus?
A Der Zeitpunkt der Defibrillation.
B Nichts Wesentliches.
C Die Postreanimationsbehandlung.
D Die reversiblen Ursachen.
E Der Zeitpunkt der Adrenalingabe.

\section{0}

Welche Therapie wäre im Rahmen der Behandlung reversibler Ursachen („4 H+HITS“) des AtemKreislauf-Stillstands bei Kindern eine Rarität?
A $1-2,5 \mathrm{ml} / \mathrm{kg}$ Glucose $20 \%$.
B $20 \mathrm{ml} / \mathrm{kg}$ einer balancierten Vollelektrolytlösung als Bolus.
C Entlastung eines Spannungspneumothorax.
D Kaliumsubstitution bei nachgewiesener Hypokaliämie.
E Rekanalisation eines verschlossenen Herzkranzgefäßes. 